\title{
Cultural Adaptation and Validation of the Malay Chronic Kidney Disease Self-Management Instrument (MCKD-SM)
}

\section{Al Sawad Ayat Ali}

Universiti Putra Malaysia

Soo Kun Lim

University of Malaya

Li Yoong Tang

University Malaya

Aneesa Abdul Rashid

Universiti Putra Malaysia

Boon-How Chew ( $\nabla$ chewboonhow@upm.edu.my)

Universiti Putra Malaysia

\section{Research Article}

Keywords: Chronic kidney disease, Chronic Kidney Disease Self-Management, Malay translation, nursing care, pre-dialysis, self-management

Posted Date: September 9th, 2021

DOI: https://doi.org/10.21203/rs.3.rs-816054/v1

License: (c) (i) This work is licensed under a Creative Commons Attribution 4.0 International License.

Read Full License 


\section{Abstract}

Background: There is growing evidence that self-management behaviour can improve outcomes for patients with chronic kidney disease (CKD). However, there are no measures available in Malay to effectively assess self-management of CKD. The aim of this study was to translate, culturally adapt, and validate the Malay Chronic Kidney Disease Self-Management (MCKD-SM) for Malay-speaking health professionals and patients.

Methods: This study was carried out in two phases: translation and cultural adaptation, and validation. Instruments were translated from English to Malay then adapted and validated in a sample of 337 patients with CKD stages 3-4 attending a nephrology clinic in a tertiary hospital in Malaysia. Construct validity was evaluated by exploratory factor analysis. Reliability of the instrument was assessed by internal consistency and test-retest reliability. The correlations between MCKD-SM and kidney disease knowledge, MCKD-SM and self-efficacy were hypothesised a priori and investigated.

Results: The Malay version of the Chronic Kidney Disease Self-Management instrument has 29 items grouped into three factors: "Understanding and Managing my CKD", "Seeking Support" and "Adherence to Recommended Regimen". The three factors accounted for $56.3 \%$ of the total variance. Each factor showed acceptable internal reliability with Cronbach's a from 0.885-0.960. 2-week intra-rater test-retest reliability intraclass correlation coefficient values for all items ranged between 0.938 to 1.000. MCKD-SM scores significantly correlates with kidney disease knowledge $(r=0.366, p<0.01)$ and self-efficacy $(r=$ $0.212, p<0.01)$.

Conclusion: The Malay version of the CKD-SM was found to be a valid and reliable patient-reported outcome measure of pre-dialysis CKD self-management behaviour in the Malay-speaking population.

\section{Background}

The growing number in incidence and prevalence of chronic kidney disease (CKD) and end stage kidney disease (ESKD) has now become a global challenge. The prevalence of CKD is projected at $11-13 \%$ across the globe [1]. Progression to advanced stage CKD is associated with increased morbidity and mortality which in turn results in decreased quality of life and premature death [2]. Progression of the CKD can be slowed down by early detection and intervention. In order to effectively delay the progression of CKD, self-management by patients is an imminent aspect in CKD management. Because of the chronic nature of CKD, patients can not just depend soley on doctors, but also need to participate actively in the CKD management [3]. According to literature, patients' health outcomes improve when they are more active in their own chronic illness treatment $[4,5]$. As a result, self-management efforts taken by CKD patients are integral to control the ongoing symptoms and undesirable sequelae of CKD. However, there are limited studies that focus on self-management strategies which address the psychological and behavioural complexities inherent in any chronic diseases and it is vital for effective management of CKD [2]. 
A viable measurement of self-management behaviour is important to measure the success of interventions designed to facilitate patients in managing their CKD. However, there are very few patientreported outcome measures have been identifed as suitable for this population. For instance, the Perceived Kidney/Dialysis Self-Management Scale has been deployed to measure perceived selfmanagement behaviour competency among CKD patients and ESKD patients receiving haemodialysis [6]. However, this patient-reported outcome measure was modified from the Perceived Medical-Condition Self-Management Scale template designed to measure self-management behaviour among HIV and diabetic patients $[7,8]$.

Due to the intricate concept of self-management, reliable and valid measures are crucial to capture accurate empirical data. The CKD Self-Management (CKD-SM) instrument is an example of such comprehensive measure that can be found in English language despite its initial development in Chinese and assessment in Taiwan [9]. The CKD-SM can be used to assess how patients self-manage their CKD and to develop more relevant, patient-centred teaching and implement interventions tailored to the needs of individual patients. The CKD-SM has been recently translated, culturally adapted, and validated in both Vietnamese and Arabic languages to enable the measurement of self-management behaviour for all CKD stages $[10,11]$. Additionally, the reliability and validity of the modified CKD-SM was assessed in Australian context as well [12].

The incidence of CKD in Malaysia is increasing at an alarming rate due to the growing burden of diabetes and hypertension and aging population [13]. The CKD prevalence in 2018 increased to 15.48\% [13] from $9.07 \%$ in 2011 [14]. Data showed a high number of CKD-related complications, as well as persistent poor disease control and management particularly among those at late stage of CKD [15]. On top of that, there is a lack of studies on evaluation of self-management behaviour among patients with early CKD, wherein special attention should be paid to those with low education level and multiple co-morbidities [15]. Given the importance of disease self-management, it is imminent to translate and validate patient-reported outcome measure to facilitate the assessment of self-management among Malay-speaking patients diagnosed with CKD. The Malay language refers to the national language of Malaysia and is spoken by the majority of its citizens.

The objective of this study is to translate the CKD-SM instrument into the Malay language, so that it is suitable, reliable, and valid to assess the self-management behaviour among Malay-speaking CKD patients.

\section{Methods}

\section{Design}

This study is part of the larger chronic kidney disease nurse-led self-management support program (CKDNLSM) study [16]. First, forward, and backward translations were conducted by a panel of experts who reviewed the translations and conducted cultural adaptation. The Malay version of CKD-SM (MCKD-SM) was subjected to psychometric assessment. 


\section{Instruments}

\section{Chronic Kidney Disease Self-Management (CKD-SM)}

The CKD-SM, which refers to a self-administered instrument, has been deployed to assess selfmanagement behaviour among CKD patients [9]. Approval to use of CKD-SM was granted by the developer. This 29-item instrument is composed of the following four factors:

Factor 1: Self-integration consists of 11 items $(7,11-12,14,18-19,22-23,25-26, \& 28)$. It examines how a patient attains a balanced life via lifestyle adjustment by incorporating the recommended treatment regimens and self-management activities.

Factor 2: Problem-solving consists of 9 items $(2,5-6,9,16,20,24,27, \& 29)$. It explores a patient's capability of seeking resources and gaining information on CKD to overcome the issues.

Factor 3: Seeking Social Support has 5 items $(1,3-4,10, \& 15)$. It examines a patient's capability in seeking support from important others to address issues related to CKD.

Factor 4: Adherence to Recommended Regimens consists of 4 items $(8,13,17, \& 21)$. It assesses if a patient follows the recommended treatment and healthcare regimens.

The four-point Likert scale was deployed for all items; 1 (never) to 4 (always). The total scores for each factor are as follows: Self-integration (11-44), Problem-solving (9-36), Seeking Social Support (5-20), and Adherence to Recommended Regimen (4-16). The overall score is between 29 and 116, whereby higher scores signify better CKD self-management behaviour. For early-stage CKD, the original English CKD-SM instrument showed good internal consistency with 0.88 score for Cronbach's alpha and good sampling adequacy with 0.89 score for Kaiser-Meyer-Olkin (KMO) [9]. A 2-week test-retest analysis of CKD-SM in early-stage CKD indicated good stability with intraclass correlation coefficient (ICC) of 0.72 [9].

\section{Setting}

This study was carried out at the Nephrology Clinic located in a tertiary teaching hospital situated in Kuala Lumpur, Malaysia. The patients there were referred by general practitioners from other healthcare centres. The clinic supports patients with early CKD right through to those who need renal replacement therapy (RRT). Patients with CKD Stages $2-5$ are managed by nephrologists and qualified CKD nurseeducators.

\section{Inclusion and exclusion criteria}

The following lists the inclusion criteria for this study:

(a) Adult with CKD stages 3-4 (defined as eGFR of $15-59 \mathrm{ml} / \mathrm{min} / 173 \mathrm{~m}^{2}$ with evidence of kidney damage) 
(b) Age $\geq 18$ years

(c) Able to understand, speak, and read the Malay language.

Additionally, the participants must not participate in cognitive debriefing, as well as not diagnosed of preexisting cognitive/vision impairment and serious illness (cancer, stroke, and dementia).

\section{Data collection and procedure}

Data collection was conducted between June 2019 and September 2020. Eligible participants were identified by researchers at the Nephrology Clinic and were recruited using consecutive sampling. After obtaining informed consent by the participants, self-administered instruments on MCKD-SM, Kidney Disease Knowledge Survey (KiKS), and Self-efficacy for Managing Chronic Disease (SEMCD) were distributed to the participants. Demographic data of the participants (gender, age, marital status, ethnicity, employment status, \& academic background) were captured in a quiet room located at the clinic. Other medical information, including CKD stages, was gathered from medical records. Re-testing was performed 2 weeks later.

\section{Translation and cultural adaptation process}

The English to Malay translation process was executed in four steps [17, 18], namely: forwardtranslation, expert panel consideration and back-translation, pre-testing and cognitive debriefing, as well as the final version (see Fig. 1). First, the original instrument was forward translated from English to Malay in an independent manner by two professional bilingual native Malay speakers from the Faculty of Languages and Linguistics, in University Malaya. Next, two initial forward translations were discussed with different points reconciled and harmonized to produce MCKD-SM. After that, this instrument was assessed in terms of idiomatic, semantic, and conceptual equivalence by 10 experts ( 1 nephrologist, 2 nursing academicians with experience in instrument validation, 2 CKD nurses, 2 family medicine specialists, \& 3 CKD patients) [19]. Both translations were reviewed by them prior to discussing in iteration and reaching a consensus on MCKD-SM instrument. Back translation from Malay to English was carried out by 2 qualified local bilingual translators from non-medical background and who were blinded to the original English versions. The translation was re-discussed by the experts to ascertain its similarity with the original instrument and the final MCKD-SM was agreed to move to the next stage.

\section{Pre-testing and cognitive debriefing}

Instrument pilot was performed using purposive sample of ten CKD Malay patients, recruited from the selected Nephrology Clinic. The participants filled in the self-administered instrument within 10-15 minutes. Next, they were requested to provide feedback on the clarity of words and sentences of the instrument, as well as its intelligibility in all aspects of the instrument. Minor changes were made, such as replacing some translated terms to more commonly used terms suggested by the patients, for example item 2: "thinking over reasons about bad laboratory data" and item 13: "Don't follow care providers' suggestion to exercise" were translated as "Memikirkan sebab-sebab mengenai data makmal 
yang teruk" and "Tidak mematuhi cadangan penyedia penjagaan untuk bersenam", respectively. Next, the patients suggested 'teruk' (bad) to be replaced with 'tidak baik' (less good), and 'penyedia penjagaan' (care providers) with 'pengamal perubatan' (medical practitioners)". The final version of the MCKD-SM was assessed for consistency and validation analysis reflected in COSMIN (COnsensus-based Standards for the selection of health Measurement INstruments) approach [20].

\section{Psychometric evaluation}

Psychometric measurement properties include convergent validity, factor analysis, internal consistency, and intra-rater test-retest reliability [16]. Exploratory factor analysis (EFA). was performed by applying the principal component extraction and Promin rotation methods. In this context, polychoric correlation method was executed, which is suitable for ordinal variables and items with the Likert-type response scale. Polychoric correlation is advised when the univariate distributions of ordinal items are asymmetric or with excess of kurtosis. The Factor 10.10 .02 program [21] was applied to conduct the EFA. Parallel analysis was performed to determine the number of factors using optimal parallel analysis (random permutation) option in the software [22]. The scree plot was used to support the parallel analysis findings.

Internal consistency was examined using Cronbach's a coefficient. Cronbach's $a<0.70$ denotes inadequate consistency, while $0.70-0.90$ signifies adequate internal consistency [23]. Intra-rater 2-week test-retest reliability was performed by estimating the ICC. The ICC of 0.70 is the minimum standard for reliability [24]. Pearson's correlation coefficient was performed to assess the correlations between kidney disease knowledge (KiKS) and self-efficacy (SEMCD) with MCKD-SM.

The KiKS measures patients' knowledge about kidney disease, especially those who need not undergo RRT [25]. This 28 items KiKS is composed of three factors that measure general knowledge on kidney disease, kidney functions, and progression symptoms. Correct response is given a score of 1 , while 0 for otherwise. With total score ranging at $0-28$, higher scores denote higher knowledge level of kidney disease.

The 6-item SEMCD measures the self-efficacy of chronic disease patients [26]. The 10-point Likert scale was employed in this instrument: 1 (not at all confident) to 10 (totally confident). With the total score ranging at 6-60, higher scores denote better self-efficacy.

It was hypothesised that MCKD-SM would correlate with KiKS and SEMCD at $~ 0.25$ [16]. Correlation coefficient scores of 0.20-0.40, 0.40-0.70, 0.70-0.90, and $>0.90$ indicate weak, moderate, strong, and very strong correlations, respectively [27].

\section{Sample size}

In light of psychometric properties analysis, the sample size had been based on the 1:10 ratio for each item $[19,24]$ signifying 290 participants were needed for this study because MCKD-SM is composed of 
29 items. After Considering 20\% incomplete responses, 363 (290/0.8) participants were invited for this study. During the 2-week intra-rater test-retest testing, 50 participants were re-invited for participation [24].

\section{Statistical analysis}

The gathered data were analysed using Statistical Package for Social Science (SPSS) version 25.0 (SPSS, Chicago, IL) and FACTOR (10.10.02) software [21]. Descriptive statistics were generated for individual item scores and demographic data. The EFA using principal component analysis (PCA), a crucial aspect in tool development, was deployed to ensure the content and number of factors in the initial items set. The KMO and Bartlett's test of Sphericity were executed as well. The retained factors were determined using the following criteria: scree plot, theoretical interpretability of the resultingfactor structure, and eigenvalues $>1$. Next, the items were chosen based on four criteria, as follows: conceptual coherence of items with individual factor, factor loading $>0.3$ [28], no cross-factor loaded items, and minimum factor membership of three items. After that, the internal consistency of MCKD-SM was determined based on Cronbach's a. Test-retest reliability was determined using ICC with receipt of completed retest instrument. As for convergent validity, Pearson correlations among CKD selfmanagement behaviour, kidney disaese knowledge and self-efficacy were ascertained as all scores displayed normal distribution.

\section{Results}

\section{Characteristics of sample}

From the 337 participants who had completed the first test, 63 of them agreed to complete retest. The participants were between 21 and 87 years old with mean score of $61.9(S D=13.1)$. Half of them were males $(n=192)$ and 230 of them were Malays $(68.2 \%)$. Most of the participants had CKD Stages 3a $(34.1 \%, n=115)$ and $3 b(40.9 \%, n=138)$. Table 1 lists their characteristics. 
Table 1

Participant characteristics $(\mathrm{N}=337)$

\begin{tabular}{|c|c|c|c|}
\hline Characteristics & Mean (SD)* & $\begin{array}{l}\text { Number } \\
(n=337)\end{array}$ & $\begin{array}{l}\text { Percentage, } \\
\%\end{array}$ \\
\hline Age (year) & $61.9(S D=13.1)$ & & \\
\hline \multicolumn{4}{|l|}{ Gender } \\
\hline Male & & 192 & 57.0 \\
\hline Female & & 145 & 43.0 \\
\hline \multicolumn{4}{|l|}{ Ethnicity } \\
\hline Malay & & 230 & 68.2 \\
\hline Chinese & & 39 & 11.6 \\
\hline Indian & & 63 & 18.7 \\
\hline Aborigines & & 1 & 0.3 \\
\hline Others & & 4 & 1.2 \\
\hline \multicolumn{4}{|l|}{ Marital status } \\
\hline Married & & 249 & 73.9 \\
\hline Unmarried & & 88 & 26.1 \\
\hline \multicolumn{4}{|l|}{ Education } \\
\hline No education & & 10 & 3.0 \\
\hline Primary school level & & 40 & 11.9 \\
\hline Secondary school level & & 163 & 48.4 \\
\hline Tertiary level & & 124 & 36.8 \\
\hline \multicolumn{4}{|l|}{ Occupation } \\
\hline Working & & 93 & 27.6 \\
\hline Not working & & 77 & 22.8 \\
\hline Retired & & 164 & 48.7 \\
\hline Student & & 3 & 0.9 \\
\hline
\end{tabular}




\begin{tabular}{|lll|}
\hline Characteristics & \multicolumn{1}{c|}{$\begin{array}{ll}\text { Mean (SD)* } \\
\text { CKD stage (eGFR mL/min/1.73 m2) }\end{array}$} & $\begin{array}{l}\text { Percentage, } \\
(\mathbf{n}=337)\end{array}$ \\
\hline Stage 3a (45-59) & 115 & 34.1 \\
Stage 3b (30-44) & 138 & 40.9 \\
Stage 4 (15-29) & 84 & 24.9 \\
\hline CKD, chronic kidney disease; eGFR, estimated glomerular filtration rate; SD, standard deviation. \\
\hline
\end{tabular}

\section{Dimensionality}

The EFA was applied to determine the factor structure among the 29 items in MCKD-SM. Several wellknown criteria for factorability of correlation were employed. First, the KMO was $0.909(>0.6)$, and the Bartlett's test of sphericity was significant $\left(\chi^{2}(406)=3735.9, p<0.001\right)$. The initial communalities are estimates of the variance in each variable accounted for by all factors, whereas small values $(<0.3)$ signify variables that fail to fit the factor solution. Turning to this present study, all initial communalities exceeded the threshold (all loading factors $>0.3$ ) [28].

When compared to the original instrument [9] that has four factors, the results of parallel analysis on all 29 items identified only three factors: Self-integration, Seeking Social Support, and Adherence to Recommended Regimen. The Problem-solving factor was absorbed and the items were distributed into Self-integration and Seeking Social Support. In addition to the original 11 items of Self-integration, 5 items $(9,20,24,27, \& 29)$ were added to Self-integration instead of Problem-solving. Similarly, in addition to the original 5 items of Seeking Social Support, 4 items $(2,5,6, \& 16)$ were added to Seeking Social Support rather than Problem-solving. Hence, Self-integration of MCKD-SM has 16 items and the factor is renamed to 'Understanding and Managing my CKD'. Similarly, Seeking Social Support in MCKD-SM has 9 items and the factor is renamed to 'Seeking Support'. Four items are retained in the Adherence to Recommended Regimen factor.

The eigenvalues and total variance explained by the three factors is presented in Table 2 and scree plot (see Fig. 2). The results after Promin rotation revealed that the first factor included 16 items with loading factor $>0.3$. Most items in this factor measure the 'Understanding and Managing my CKD' and explained $31.9 \%$ of total variance. The second factor consist of nine items related to 'Seeking Support' and explained $15.1 \%$ of total variance. The last factor has four items related to 'Adherence to Recommended Regimen' and explained $9.3 \%$ of total variance. Total variance explained by these three factors was $56.3 \%$, which exceeded the recommended value of $50 \%$ as the general rule [29]. 
Table 2

Factor loading and Cronbach's alpha for the 29-item MCKD-SM

\section{Factor Loading}

Items

$\begin{array}{lll}\text { Factor } & \text { Factor } & \text { Factor } \\ 1 & 2 & 3\end{array}$

Factor 1: Understanding and Managing my CKD

26. Adjusting lifestyle to maintain the best condition

0.865

20. ${ }^{\circledR}$ Actively understanding risk factors of CKD

0.859

19. Giving up bad habits harmful for kidney

22. Managing food portions and choices in social activity

0.840

12. Heeding habits that may affect kidney function

0.831

18. Managing CKD to stay healthy

0.824

25. Managing food followed to care providers' suggestion

0.800

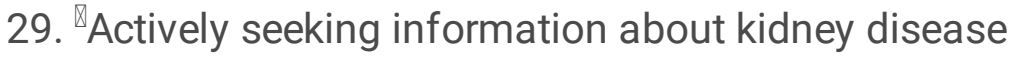

0.753

23. Adjusting CKD care to fit new situation

0.721

14. Changing lifestyle to avoid worse of kidney function

0.705

11. Merging CKD management into daily life

0.699

7. Managing food to avoid harm for kidney

0.697

24. ${ }^{\circledR}$ Finding out reasons for signs and symptoms

0.664

27. Utilizing different ways to clarify questions about treatment

0.588 plan

28. Participating selectively in social activities

9. 『Utilizing different ways to solve problems

0.450

Factor 2: Seeking Support

3. Telling family or friends about treatment plan

0.790

4. Sharing experience with other patients

0.753

15. Asking family or friends for help when helpless or frustrated

0.748

1. Discussing with family or friends while questioning or worrying

0.681

2. ${ }^{\text {TT}}$ Thinking over reasons about bad laboratory data 0.656

10. Sharing helpless and frustrated feeling with other patients 0.639 


\section{Factor Loading}

6. ${ }^{\mathbb{P}}$ Finding out possible reasons about high BP value

0.552

5. ${ }^{\mathbb{A}}$ Actively understanding the meaning of laboratory data

16. ${ }^{\mathbb{A}}$ Actively seeking resources to better control

Factor 3: Adherence to Recommended Regimen

21. Don't follow care providers' suggestion to control weigh

17. Don't follow care providers' suggestion to adjust diet habit

13. Don't follow care providers' suggestion to exercise

8. Don't follow the dieticians' suggestion to choose food 0.674

Eigenvalue 9.258

4.376

2.705

$\%$ Of Variance

31.9

15.1

9.3

Cronbach a

0.960

0.899

0.885

${ }^{\square}$ Originally is Problem-solving; CKD, chronic kidney disease; Understanding and Managing my CKD originally is Self-integration; Seeking Support originally is Seeking Social Support.

\section{Reliability}

The internal consistency of sub-scale was very good with Cronbach's a ranging at $0.885-0.96$. The 2week intra-rater test-retest reliability of MCKD-SM was also very good with ICC values for all items ranging at 0.938-1.000.

\section{Construct Validity (Convergent Validity)}

A positive link was noted between MCKD-SM and KiKS scores $(r=0.366, p<0.01)$, which signified that high-level self-management behaviour was related to high-level kidney disaese knowledge. Next, the positive link of MCKD-SM with SEMCD scores $(r=0.212, p<0.01)$ showed that high-level self-efficacy was related to high-level self-management behaviour.

\section{Discussion}

The MCKD-SM appeared to be as valid and reliable instrument to examine self-management behaviour among CKD Malay-speaking patients. The MCKD-SM had successfully measured crucial skills and daily activities for self-management. MCKD-SM was comprehensible to those with poor proficiency. The process of completing the survey took only 10-15 minutes. The translation and adaptation processes 
involved to yield the MCKD-SM adhered to guidelines for cross-cultural adaption of self-report measures $[17,18]$. Slight variances were noted in linguistic usage, in which the necessary grammatical and cultural amendments were made. Exceptional content validity for MCKD-SM was verified by the selected experts with vast experience in caring for CKD patients and familiar with the medical terms Inclusion of three CKD patients with varying backgrounds and work experiences ensured that the instrument content was comprehensible, thus increasing the likelihood that the participants to provide accurate responses. Referring to the COSMIN checklist, MCKD-SM displayed acceptable psychometric properties with good construct validity [20]. The COSMIN checklist evaluates the methodological quality of a study on measuring properties of health-related patient-reported outcome instrument, but not to assess the quality of the health-related patient-reported outcome instrument itself [30]. The checklist was developed with the participation of many experts in the field [30].

Problem-solving was excluded as a factor in MCKD-SM which differs from the original, Vietnamese, and Australian versions $[10,12]$. This factor (9 items) was dispersed into Understanding and Managing my CKD (originally known as Self-integration) (5 items) and Seeking Support (originally Seeking Social Support) (4 items). This is related to the local family dynamic where no individual (health) problem should be kept from the family [31, 32]. Problem-solving in health problems is a family issue, and begins in personal understanding of the problem at hands. This is clearly seen in item 20 ("Actively understanding risk factors of CKD") and 29 ("Actively seeking information about kidney disease") that recorded high scores in Cronbach's alpha (0.86 and 0.75, respectively) in Understanding and Managing my CKD factor. Seeking Social Support factor was renamed to Seeking Support due to the inclusion of Problem-solving items that broadened the support scope to professional support. This is because; patients seek clarification and meanings of laboratory results, as well as appropriate actions to better control their diseases [32].

Similar to the original $(a=0.77-0.92)$ [9], Vietnamese $(a=0.77-0.90)$ [10], Arabic $(a=0.71-0.83)$ [11], and Australian ( $\alpha=0.72-0.85)$ [12] versions, Cronbach's $a$ for all factors in MCKD-SM are reasonably high and reliable to measure self-management behaviour in the Malay-speaking population. The result of test-retest analyses showed that the MCKD-SM was very stable over the 2-week period. Besides, ICC score was higher than the original [9], Vietnamese [10], Arabic [11], and Australian versions [12]. Nonetheless, the percentage of variance in CKD self-management explained by MCKD-SM was not high (56.3\%). This is ascribed to the participants who were in relatively early CKD stages, thus many items in the instrument could have appeared less relevant than those with more advanced CKD stages. This is indicated by the $<10 \%$ of variance in the Adherence to Recommended Regimen factor, where healthy diet and exercises might not be immediately relevant to the functiong kidneys at CKD stages 3-4.

In this stduy,convergent validity was ascertained by assessing the correlations among kidney disease knowledge, self-efficacy and CKD self-management behaviour. High-level kidney disease knowledge improved CKD self-management behaviour, which is in line with past findings [33, 34]. Better self-efficacy was positivly correlated with CKD self-management behaviour, which is in agreement with prior findings $[12,35-37]$. Perhaps, the weaker than expected correlation between CKD self-management behaviour and 
SEMCD is because that the construct of self-management measured by MCKD-SM has multiple determinants not limited to kidney disease specific knowledge and self-efficacy skills [37]. According to Lai [38], age, disease duration, education and depression are other determinants that can affect one's selfefficacy and self-management in light of pre-dialysis CKD. Younger age, longer disease duration, and higher education levels are positively and independently correlated with high-level of self-management and self-efficacy. Depression is adversely correlated with self-management and self-efficacy [38]. Similarly, this study outcome revealed that improving generic self-efficacy (SEMCD), such as in symptoms control, sufficient social roles function, healthy emotional functionings, and effective communication with doctors, ia a potential facilitator to CKD self-management.

This study verifies the reliability, as well as contentand construct validity of MCKD-SM. Essentially, MCKDSM should be assessed for its applicability among other patient populations of different societal strata; due to the varying cultural, linguistic, healthcare system, healthcare provider and patient expectations, as well as self-management implementation.

\section{Strength And Limitations}

Standard translation with rigorous adaptation procedures by a panel of experts, including CKD patients, generated the MCKD-SM with exceptional cross-cultural validity. The other strength refers to the involvement of participants with a range of ages, CKD stages 3-4, and EFA-enabling sample size [39]. The correlation with the KiKS and SEMCD has supported the potential effects on CKD self-management behaviour from kidney disease knowledge and self-efficacy.

This study has some limitations. First, recruiting from one centre may limit its generalisability to other settings, such as primary care or among those from the lower socio-economic areas. Second, due to the inclusion criteria, the outcomes are less applicable to Chinese and Indians residing in Malaysia, or among those who are less proficient in the Malay language, and to those with CKD stages other than 3 and 4 . Hence, future studies should look into validating the MCKD-SM in patient groups of different ethnicities, socio-economic background and CKD stages to confirm its validity in the measuring CKD selfmanagement behaviour.

\section{Conclusion}

The MCKD-SM is a valid, reliable, and feasible patient-reported outcome measure of self-management behaviour in patients with pre-dialysis CKD. This three-factor instrument that measures CKD selfmanagement behaviour is suitable for clinical practices. Moreover, CKD nurses may deploy it as an assessment instrument when supporting or educating patients during earlier CKD stages, especially regarding understanding and managing CKD, seeking social and professional support, adherence to medications and lifestyle modifications.

\section{Abbreviations}


CKD

Chronic kidney disease; CKD-SM:Chronic kidney disease self-management; EFA:Exploratory factor analysis; eGFR:Glomerular filtration rate; ESKD:end stage kidney disease; ICC:Intraclass correlation coefficient; KiKS:Kidney Disease Knowledge Survey; KMO:Kaiser-Meyer-Olkin; MCKD-SM:Malay chronic kidney disease self-management; PCA:Principal component analysis; RRT:Renal replacement therapy; SEMCD:Self-efficacy for Managing Chronic Disease.

\section{Declarations}

\section{Ethics approval and consent to participate}

This study was approved by the Medical Research Ethics Committee of the University Malaya Medical Centre (MREC ID NO: 201952-7389) and Universiti Putra Malaysia (JKEUPM-2019-374). Written informed consent was obtained from all participants who agreed to participate. All data was kept confidential and was used for research purposes only. All methods were performed in accordance with the relevant guidelines and regulations.

\section{Consent for publication}

Not applicable.

\section{Availability of data and materials}

The datasets used and/or analyzed during the current study are available from the corresponding author on reasonable request.

\section{Competing interests}

The authors have no conflicts of interest to declare.

\section{Funding}

This research was financially supported by Technological Horizons for Compound Fertilizer Factory (C.R.2062021134), Kingdom of Saudi Arabia, Dammam; P.O.BOX 184; Post-code 31972.Tel: +966 1385 60281- Fax: +966 1385 07111. The funder has no roles in the study conception, data collection, data analysis, results interpretation and reporting.

\section{Authors' contributions}

A.S.A. drafted, formulated, and submitted the manuscript. All authors S.K., L.Y., A.A. and B.H. contributed to the study designs, read, revised, and approved the research critically for important intellectual content and helped to draft the final manuscript. All authors gave final approval of this manuscript to be published. Authorship eligibility is in accordance with the International Committee of Medical Journal Editors (ICMJE) guidelines. 


\section{Acknowledgements}

We would like to thank the management and patients at the University Malaya Medical Centre, for their support and cooperation during this study. We extend our gratitude to the CKD nurse-educators Miss. Karina Binti Abdul Wahid Pereira and Miss. Fatimah Hasanah Binti Mohd Radzi for their assistance in reviewing the translation and data collection.

\section{Authors' information}

${ }^{1}$ Department of Family Medicine, Faculty of Medicine and Health Sciences, Universiti Putra Malaysia, Selangor, Malaysia. ${ }^{2}$ Department of Nursing, King Fahd Hospital of the University (KFHU), Imam Abdulrahman Bin Faisal University (IAU), Dammam, Saudi Arabia. ${ }^{3}$ Department of Medicine (Nephrology), Faculty of Medicine, University of Malaya, Kuala Lumpur, Malaysia. ${ }^{4}$ Department of Nursing Science, Faculty of Medicine, University Malaya, Kuala Lumpur, Malaysia. ${ }^{5}$ Clinical Research Unit, Hospital Pengajar Universiti Putra Malaysia (HPUPM Teaching Hospital), Serdang, Malaysia.

\section{References}

1. N. R. Hill et al., "Global prevalence of chronic kidney disease-a systematic review and metaanalysis," PloS one, vol. 11, no. 7, pp. e0158765, 2016.

2. S. H. Phua et al., "Evaluation of self management behavior of chronic kidney disease patients," $J$ Pharm Pharmacol, vol. 5, pp. 179-188, 2017.

3. M. M. Funnell, and R. M. Anderson, "The problem with compliance in diabetes," JaMa, vol. 284, no. 13, pp. 1709-1709, 2000.

4. T. Bodenheimer, E. H. Wagner, and K. Grumbach, "Improving primary care for patients with chronic illness: the chronic care model, Part 2," Jama, vol. 288, no. 15, pp. 1909-1914, 2002.

5. M. Heisler et al., "How well do patients' assessments of their diabetes self-management correlate with actual glycemic control and receipt of recommended diabetes services?," Diabetes care, vol. 26, no. 3, pp. 738-743, 2003.

6. M. G. Wild et al., "The Perceived Medical Condition Self-Management Scale can be applied to patients with chronic kidney disease," Kidney international, vol. 92, no. 4, pp. 972-978, 2017.

7. K. A. Wallston, R. L. Rothman, and A. Cherrington, "Psychometric properties of the perceived diabetes self-management scale (PDSMS)," Journal of behavioral medicine, vol. 30, no. 5, pp. 395-401, 2007.

8. K. A. Wallston et al., "The perceived medical condition self-management scale applied to persons with HIV/AIDS," Journal of health psychology, vol. 16, no. 1, pp. 109-115, 2011.

9. C. C. Lin et al., "Psychometric evaluation of a new instrument to measure disease self-management of the early stage chronic kidney disease patients," Journal of clinical nursing, vol. 22, no. 7-8, pp. 1073-1079, 2013. 
10. N. T. Nguyen, C. Douglas, and A. Bonner, "Psychometric evaluation of the culturally and linguistically translated Vietnamese chronic kidney disease self-management instrument," International journal of nursing practice, pp. e12727, 2019.

11. H. Almutary, and N. Tayyib, "Translation, Adaptation, and Validation of Chronic Kidney Disease SelfManagement and Knowledge Instruments for People at Pre-Dialysis Stage in the Arab World," Clinical Nursing Research, pp. 10547738211006242, 2021.

12. C. Wembenyui, C. Douglas, and A. Bonner, "Validation of the Australian version of the Chronic Kidney Disease Self-Management instrument," International Journal of Nursing Practice, vol. 27, no. 2, pp. e12857, 2021.

13. T. A. Saminathan et al., "Prevalence of chronic kidney disease and its associated factors in Malaysia; findings from a nationwide population-based cross-sectional study," BMC nephrology, vol. 21, no. 1, pp. 1-11, 2020.

14. L. S. Hooi et al., "A population-based study measuring the prevalence of chronic kidney disease among adults in West Malaysia," Kidney international, vol. 84, no. 5, pp. 1034-1040, 2013.

15. S. H. Phua et al., "Evaluation of Self Management Behavior of Chronic Kidney Disease Patients," Journal of Pharmacy and Pharmacology, vol. 5, pp. 179-188, 2017.

16. A. S. Ayat Ali et al., "The effectiveness of nurse-led self-management support program for people with chronic kidney disease stage 3-4 (CKD-NLSM): Study protocol for a randomized controlled trial," Sci Prog, vol. 104, no. 2, pp. 368504211026159, Apr-Jun, 2021.

17. J. Epstein, R. M. Santo, and F. Guillemin, "A review of guidelines for cross-cultural adaptation of questionnaires could not bring out a consensus," Journal of clinical epidemiology, vol. 68, no. 4, pp. 435-441, 2015.

18. D. Wild et al., "Principles of good practice for the translation and cultural adaptation process for patient-reported outcomes (PRO) measures: report of the ISPOR Task Force for Translation and Cultural Adaptation," Value in health, vol. 8, no. 2, pp. 94-104, 2005.

19. V. D. Sousa, and W. Rojjanasrirat, "Translation, adaptation and validation of instruments or scales for use in cross-cultural health care research: a clear and user-friendly guideline," Journal of evaluation in clinical practice, vol. 17, no. 2, pp. 268-274, 2011.

20. L. B. Mokkink et al., "The COSMIN checklist for evaluating the methodological quality of studies on measurement properties: a clarification of its content," BMC medical research methodology, vol. 10, no. 1, pp. 22, 2010.

21. U. Lorenzo-Seva, and P. J. Ferrando, "FACTOR: A computer program to fit the exploratory factor analysis model," Behavior research methods, vol. 38, no. 1, pp. 88-91, 2006.

22. M. E. Timmerman, and U. Lorenzo-Seva, "Dimensionality assessment of ordered polytomous items with parallel analysis," Psychological methods, vol. 16, no. 2, pp. 209, 2011.

23. J. Pallant, SPSS survival manual: A step by step guide to data analysis using IBM SPSS. Routledge, 2020. 
24. C. B. Terwee et al., "Quality criteria were proposed for measurement properties of health status questionnaires," Journal of clinical epidemiology, vol. 60, no. 1, pp. 34-42, 2007.

25. J. A. Wright et al., "Development and results of a kidney disease knowledge survey given to patients with CKD," American journal of kidney diseases, vol. 57, no. 3, pp. 387-395, 2011.

26. K. R. Lorig et al., "Effect of a self-management program on patients with chronic disease," Effective clinical practice: ECP, vol. 4, no. 6, pp. 256-262, 2001.

27. B. R. Overholser, and K. M. Sowinski, "Biostatistics primer: part 2," Nutrition in Clinical Practice, vol. 23, no. 1, pp. 76-84, 2008.

28. P. Samuels, "Advice on exploratory factor analysis," 2017.

29. D. L. Streiner, "10 Figuring Out Factors: The Use and Misuse of Factor Analysis," A Guide for the Statistically Perplexed, pp. 110-122: University of Toronto Press, 2020.

30. L. B. Mokkink et al., "The COSMIN checklist for assessing the methodological quality of studies on measurement properties of health status measurement instruments: an international Delphi study," Quality of life research, vol. 19, no. 4, pp. 539-549, 2010.

31. H. Yatim et al., "Factors influencing patients' hypertension self-management and sustainable selfcare practices: a qualitative study," Public health, vol. 173, pp. 5-8, 2019.

32. A. Abdullah et al., "Health literacy experiences of multi-ethnic patients and their health-care providers in the management of type 2 diabetes in Malaysia: a qualitative study," Health Expectations, vol. 23, no. 5, pp. 1166-1176, 2020.

33. R. Devraj, and E. J. Gordon, "Health literacy and kidney disease: toward a new line of research," American journal of kidney diseases, vol. 53, no. 5, pp. 884-889, 2009.

34. C. D. Enworom, and M. Tabi, "Evaluation of kidney disease education on clinical outcomes and knowledge of self-management behaviors of patients with chronic kidney disease," Nephrol Nurs J, vol. 42, no. 4, pp. 363-373, 2015.

35. R. B. Curtin et al., "Self-efficacy and self-management behaviors in patients with chronic kidney disease," Advances in chronic kidney disease, vol. 15, no. 2, pp. 191-205, 2008.

36. C.-C. Lin et al., "The chronic kidney disease self-efficacy (CKD-SE) instrument: development and psychometric evaluation," Nephrology Dialysis Transplantation, vol. 27, no. 10, pp. 3828-3834, 2012.

37. N. T. Nguyen, C. Douglas, and A. Bonner, "Effectiveness of self-management programme in people with chronic kidney disease: A pragmatic randomized controlled trial," Journal of advanced nursing, vol. 75, no. 3, pp. 652-664, 2019.

38. P.-C. Lai et al., "Factors influencing self-efficacy and self-management among patients with pre-endstage renal disease (Pre-esrd)." p. 266.

39. A. L. Comrey, and H. B. Lee, "A First Course in Factor Analysis, 2nd Edn. Hillsdale, NJ: L," Erlbaum Associates, 1992.

\section{Figures}




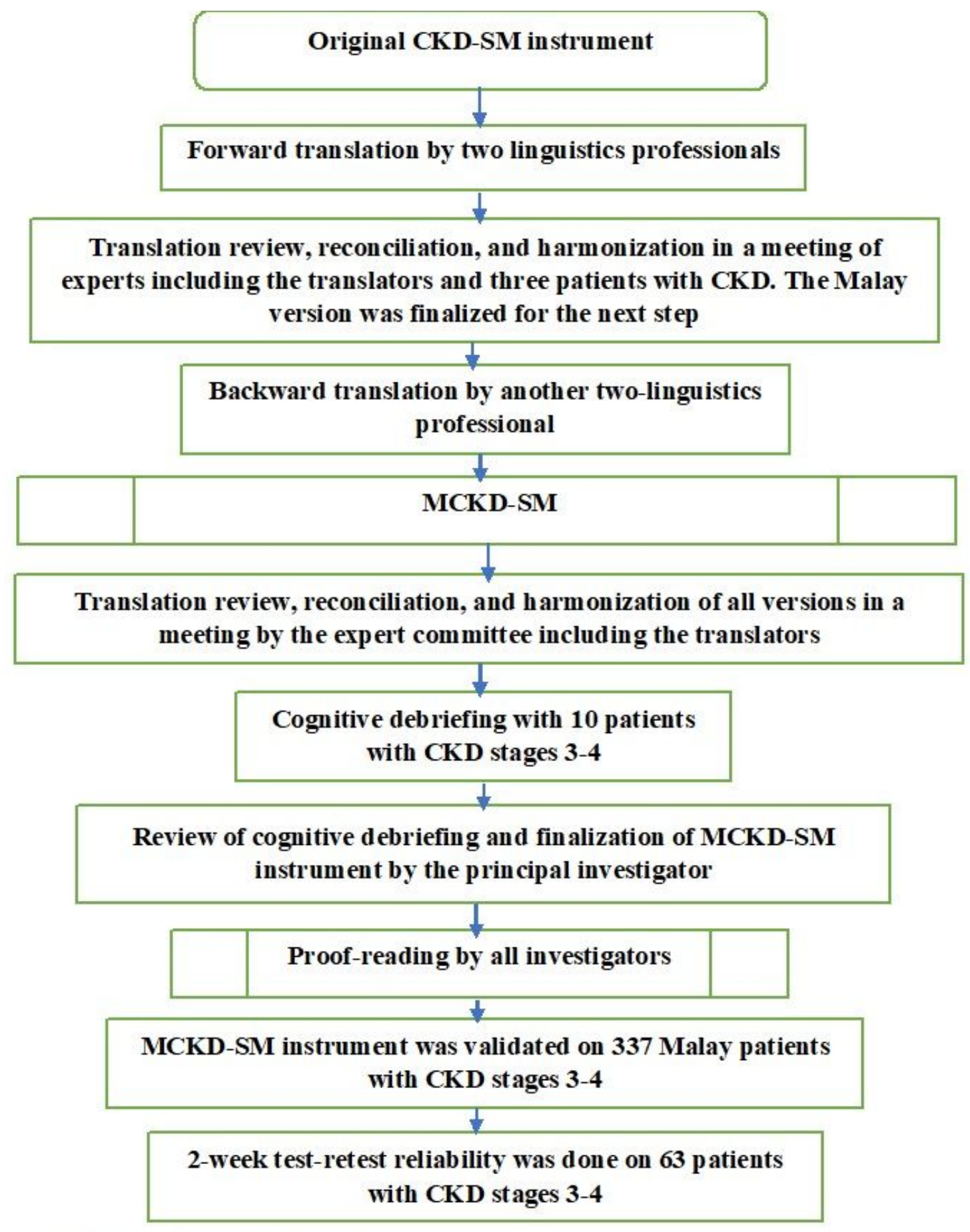

Fig. 1 Translation and valid ation process

CKD, chronic kidney disease; CKD-SM, Chronic Kidney Disease Self-m an agement; MCKD-SM, Malay Chronic Kidney Disease Self-man agement.

Figure 1

Translation and validation process 


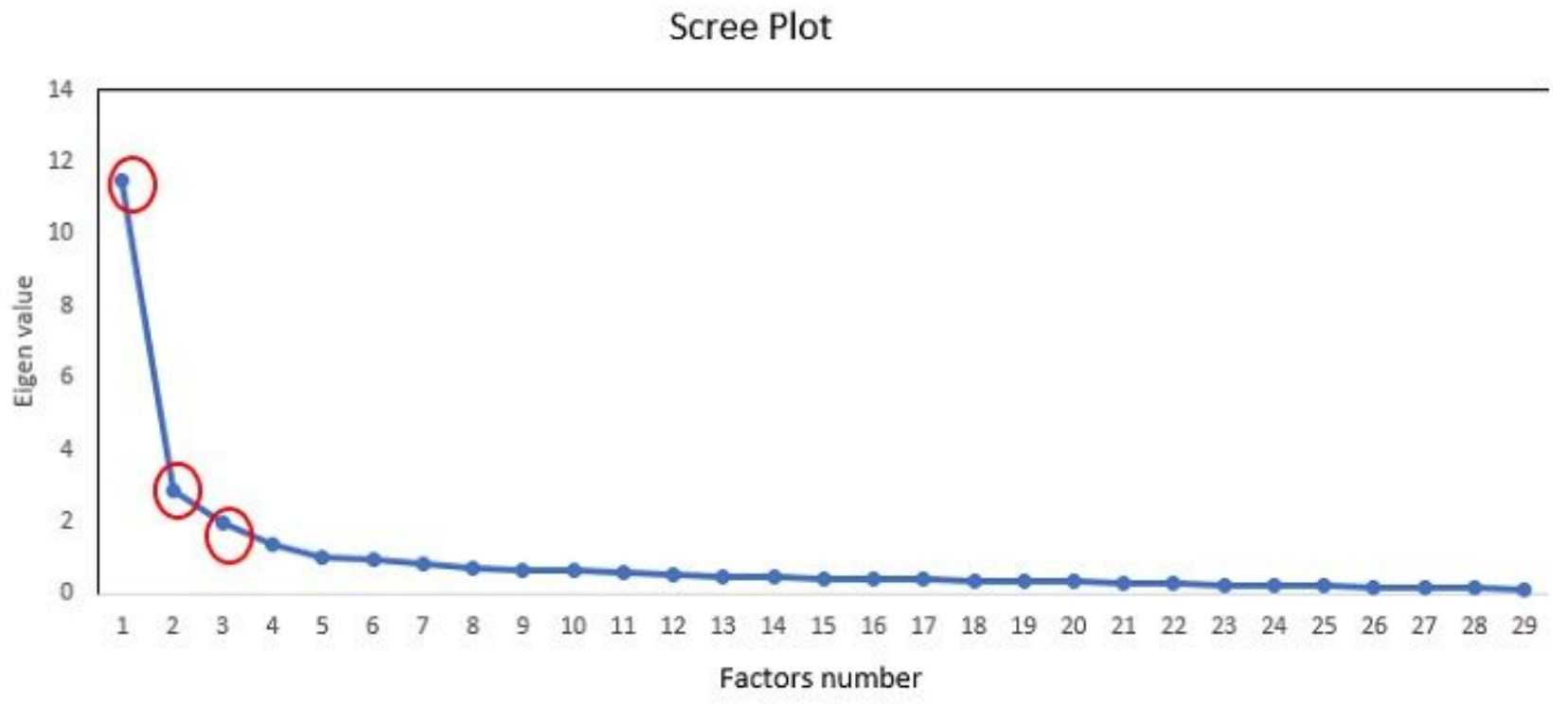

Fig. 2 Scree plot for exploratory factor analysis on the Malay Chronic Kidney Disease SelfManagement (MCKD-SM).

\section{Figure 2}

Scree plot for exploratory factor analysis on the Malay Chronic Kidney Disease Self- Management (MCKD-SM). 